\title{
Percepção de enfermeiros de unidades de internação clínica sobre a Sistematização da Assistência de Enfermagem
}

RESUMO | Objetivo: identificar a percepção dos enfermeiros de unidades de internação clínica sobre a importância da Sistematização da Assistência de Enfermagem para o planejamento da assistência. Método: Estudo descritivo, qualitativo, realizado após aprovação do comitê de ética em pesquisa, em um hospital universitário de Porto Alegre. Resultados: foram entrevistados 18 enfermeiros. A análise temática dos dados revelou as seguintes áreas: Sistematização da Assistência de Enfermagem: Conhecimento e Aplicação na prática profissional: dificuldades encontradas. Conclusão: apesar das comprovações científicas de sua eficiência, a implementação da SAE necessita superar as limitações impostas pela falta de compreensão do papel do enfermeiro em sua prática assistencial. $\mathrm{O}$ aprimoramento científico torna-se imperativo no desenvolvimento de atitudes e habilidades direcionadas a um cuidado ético e responsável.

Palavras-chaves: Enfermagem; Processo de Enfermagem; Prática Profissional.

\begin{abstract}
Objective: identify the perception of the nurses of clinical hospitalization units about the importance of Nursing Care Systematization for care planning. Method: Descriptive, qualitative study, carried out after approval of the research ethics committee, at a university hospital in Porto Alegre. Results: 18 nurses were interviewed. The thematic analysis of the data revealed the following areas: Systematization of Nursing Assistance: Knowledge and Application in professional practice: difficulties encountered. Conclusion: despite the scientific evidence of its efficiency, the SAE implementation needs to overcome the limitations imposed by the lack of understanding of the role of nurses in their care practice. The scientific improvement becomes imperative in the development of attitudes and abilities directed towards an ethical and responsible care.
\end{abstract}

Keywords: Nursing; Nursing Process; Professional Practice.

RESUMEN | Objetivo: identificar la percepción de las enfermeras de las unidades de hospitalización clínica sobre la importancia de la sistematización de la atención de enfermería para la planificación de la atención. Método: Estudio descriptivo, cualitativo, realizado después de la aprobación del comité de ética de la investigación, en un hospital universitario de Porto Alegre. Resultados: se entrevistó a 18 enfermeras. El análisis temático de los datos reveló las siguientes áreas: Sistematización de la asistencia de enfermería: conocimiento y aplicación en la práctica profesional: dificultades encontradas. Conclusión: a pesar de la evidencia científica de su eficiencia, la implementación de SAE debe superar las limitaciones impuestas por la falta de comprensión del papel de las enfermeras en su práctica de atención. La mejora científica se vuelve imperativa en el desarrollo de actitudes y habilidades dirigidas hacia un cuidado ético y responsable.

Descriptores: Enfermería; Proceso de Enfermeira; Práctica Profesional.

\section{Jéssica Pereira da Cunha Machado}

Enfermeira. Universidade Luterana do Brasil (ULBRA), Gravataí, RS.

\section{Débora Monteiro da Silva}

Enfermeira. Mestre em Enfermagem. Professora do curso de graduação em enfermagem da Universidade Luterana do Brasil (ULBRA), Gravataí, RS.

\section{Elisangela Souza}

Enfermeira. Especialista. Membro da Comissão do Processo de Enfermagem do Hospital de Clinicas de Porto Alegre/RS.

\section{Cecília Drebes Pedron}

Enfermeira. Doutora em enfermagem pela UFRGS. Professora adjunta do curso de enfermagem do Departamento MaternoInfantil da UFRGS.

\section{Cristiane Helena Gallasch}

Enfermeira. Doutora em Enfermagem. Professora Adjunta da Faculdade de Enfermagem da Universidade do Estado do Rio de Janeiro. Rio de Janeiro, RJ.

\section{Priscila Cristina da Silva Thiengo}

Enfermeira. Doutoranda em Enfermagem. Professora Assistente da Faculdade de Enfermagem da Universidade do Estado do Rio de Janeiro. Rio de Janeiro, RJ.

Recebido em: 30/05/2019 Aprovado em: 30/05/2019

\section{INTRODUÇÃO}

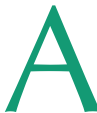
Sistematização da Assistência de Enfermagem (SAE) é um tema presente nas discussões contemporâneas, não somente com objetivo de atender as responsabilidades legais de sua prática, mas principalmente por benefícios relatados através de sua aplicação, incluindo a qualificação da assistência prestada. Com o embasamento científico do Processo de Enfermagem (PE) nas teorias da profissão, este processo é altamente discutido pelos profissionais da área por sua importância e aplicabilidade na prática profissional(1).

Historicamente, percebe-se a evolução da enfermagem em três momentos: o primeiro com Florence Nightin- 
gale ao incitar a profissão nos moldes modernos; o segundo na construção das teorias de Enfermagem, com intuito de solidificar a profissão cientificamente; e o terceiro que corresponde à estruturação SAE, presente até a atualidade $^{(2)}$.

A SAE tem sido utilizada nas instituições de saúde como uma metodologia de assistência por meio do PE. Este, por sua vez, vem sendo implantado no Brasil desde a década de 1970 por Wanda de Aguiar Horta. No entanto, somente em 2002 a SAE recebeu o apoio legal do Conselho Federal de Enfermagem (COFEN) pela Resolução n. ${ }^{\circ} 272$, a fim de ser implementada em todas as instituições de saúde do Brasil ${ }^{(3)}$, como atividade privativa do enfermeiro que utiliza método e estratégia de trabalho científico para a identificação das situações de saúde e doença, subsidiando as ações da assistência de enfermagem que possam contribuir para a promoção, prevenção, recuperação e reabilitação da saúde do indivíduo, família e comunidade, partilhando seus conhecimentos no contexto do trabalho multiprofissional $^{(4,5)}$.

Com foco centrado no paciente, o PE desenvolve-se por meio do olhar observador do enfermeiro, compondo-se das seguintes fases: investigação, que exige conhecimento e comprometimento teórico-prático-metodológico diagnóstico de enfermagem, a partir do julgamento clínico, elaborando o planejamento e as intervenções que favorecerão a organização do cuidado; planejamento, realizado continuamente a partir da elaboração de uma trajetória que busque a recuperação e manutenção da saúde; implementação, associada ao planejamento, realizada por meio da dinâmica apontada pelos diagnósticos, com direcionamento biopsicossocial, cultural, ambiental e espiritual envolvendo o ambiente de intervenção assistencial no cuidado; e avaliação, que fornece dados sobre os resultados das ações, exigindo ha- bilidade, conhecimento e articulação científica para traçar estratégias ao alcance dos resultados esperados, de forma segura ${ }^{(6-8)}$.

Ressalta-se que os registros de enfermagem, além de cumprir uma exigência legal, contribuem com informações para organizar o trabalho da enfermagem, com o propósito de disponibilizar informações do serviço prestado e promover a comunicação entre os integrantes da equipe ${ }^{(7)}$.

A discussão a respeito da SAE é mandatória, uma vez que as tentativas de implementação muitas vezes não atingem o objetivo esperado, devido a dificuldades das instituições no processo de implementação, bem como relutância dos próprios profissionais de enfermagem, tornando-se basicamente uma atividade burocrática. É que seja observada e analisada pela equipe de enfermagem, sendo implantada com conhecimento e metas possíveis de serem alcançadas, a fim de uma assistência qualificada, por meio de um trabalho de enfermagem eficiente com resultados positivos ${ }^{(3)}$.

A partir do contexto apresentado, questiona-se: Como os enfermeiros de unidades de internação percebem a importância da Sistematização da Assistência de Enfermagem?

Assim, o presente estudo objetiva identificar a percepção dos enfermeiros de unidades de internação clínica sobre a importância da Sistematização da Assistência de Enfermagem para o planejamento da assistência.

\section{METODOLOGIA}

Trata-se de um estudo descritivo, com abordagem qualitativa, realizado em hospital universitário público de grande porte, localizado na região metropolitana de Porto Alegre, RS, entre março e junho de 2015, onde atuavam os 18 enfermeiros participantes, em diferentes turnos de trabalho.

Estabeleceu-se como critério de inclusão enfermeiros em atividades assistenciais e de exclusão o afastamento do profissional por motivo de licenças e férias. A recusa em participar do estudo foi considerada como possibilidade de perda de participantes.

A coleta de dados ocorreu por meio de entrevista semiestruturada, realizada em local apropriado e indicado pelo participante, com gravação em mídia eletrônica (MP4). As informações coletadas incluíram dados sobre conhecimento, compreensão, utilização, dificuldades, vantagens e desvantagens da SAE. Além disso, os participantes foram caracterizados a partir da idade, sexo, local de trabaIho, titulação acadêmica/pós-graduação e tempo de formação. Foi estabelecido o critério de saturação dos dados, que significa amostrar até o ponto em que não se obtém nenhuma nova informação e é atingida a redundância ${ }^{(9)}$.

A análise dos dados foi realizada a partir do referencial de Análise Temática, com a proposta de descobrir comportamento e sentido nas falas dos entrevistados, por meio de três etapas: transcrição do material eletrônico, a releitura e a ordenação do material quanto a sua relevância ${ }^{(10)}$. Após a análise, obtiveram-se áreas temáticas: Sistematização da Assistência de Enfermagem: Conhecimento e Aplicação na prática profissional: dificuldades encontradas.

O estudo obteve autorização da chefia local, assim como aprovação do Comitê de Ética em Pesquisa da instituição, sob protocolo n. ${ }^{\circ} 1002707$ e CAAE 4292.5015.1.000.5349, obedecendo todos os preceitos éticos incluídos na legislação vigente ${ }^{(11)}$.

\section{RESULTADOS E DISCUSSÃO}

Os 18 enfermeiros entrevistados encontravam-se na faixa etária entre 22 a 49 anos, sendo 95,5\% $(n=17)$ do sexo feminino, $83,3 \%(n=15)$ com dois 
a 60 meses de experiência profissional, $88,9 \%(n=16)$ egressos de instituições públicas e $61,1 \%(n=11)$ com pós-graduação na modalidade especialização concluída ou em andamento.

Corroborando com a pesquisa nacional do perfil de enfermagem, houve maior prevalência de participantes, do sexo feminino, apesar do reconhecido aumento da entrada de homens na profissão. Verifica-se, ainda, que os mesmos encontram-se, em sua maioria, nas fases de "Início da vida profissional" e "Formação profissional" o que se relaciona diretamente com a busca por qualificação, por meio de cursos de especialização ${ }^{(12)}$. Ressalta-se que, para implementação e continuidade da SAE, é essencial o aprimoramento técnico-científico do enfermeiro ${ }^{(13)}$.

Após análise dos dados, foram identificadas duas áreas temáticas: Sistematização da Assistência de Enfermagem: Conhecimento e Aplicação na prática profissional: dificuldades encontradas para melhor discutir os resultados obtidos.

Sistematização da Assistência de Enfermagem: Conhecimento

Quando questionados sobre o entendimento e o significado da SAE, evidenciou-se que os entrevistados possuem uma conceituação científica pouco consistente, apresentando-se confusos e inseguros, como demonstrado nas falas a seguir:

"Eu acho que a assistência de enfermagem é mais para facilitar o nosso dia a dia né, pras coisas andarem mais fácil (...) eu acho que quando a gente consegue realmente aplicar funciona (...) as coisas realmente facilitam. É tão automático (...) a gente às vezes nem vê o que é que tu faz que é da sistematização o que não é" (E5).

"Sistematização eu acredito que é o processo da tua atuação do enfermeiro dentro do local e os processos que ele tem que realizar (...) todas as atividades que tem que realizar (...) toda equipe funciona junto, toda demanda que tu atende (...) no caso assim, eu enxergo como tu divide, organiza o teu dia a dia, o teu trabalho e as hierarquias existentes dentro dessa funcionalidade do trabalho, e como que é o principal ponto tu que faz o teu dia funcionar, dentro da instituição dentro das regras e normativas da instituição que tu trabalha também." (E7).

A complexidade de propor um cuidado sistematizado e planejado deve superar as atitudes empíricas e progredir para a cientificidade. Todavia, percebe-se na prática algumas dificuldades para aplicação da SAE e, até mesmo, a não aplicabilidade. Nesse sentido, as tomadas de decisão e as ações concentram-se somente no senso comum, não havendo planejamento e, consequentemente, dificultando o raciocínio lógico para o desenvolvimento de ações que poderiam ser estudadas, discutidas e comprovadas na ciência da enfermagem ${ }^{(14)}$.

Alguns enfermeiros, embora tenham respondido de forma incompleta, conseguiram descrever que a SAE faz parte do cuidado prestado.

"Na verdade, eu entendo como uma padronização, uma qualificação no atendimento dado ao paciente, é um complemento para nossa rotina, é sempre buscar melhorar o atendimento ao paciente". (E15).

"[...] A sistematização vem para te ajudar. Te dá trabalho, mas vem pra te ajudar, pra organizar o teu serviço e pra dar uma valorização também se tu focar no que tu encontrou. A gente tem o hábito de fazer e fazer e não registrar. A gente faz horrores de coisa. No momento que tu senta e tu começa a colocar aquilo no papel teu trabalho aparece e tu começa a te sentir mais valorizado, mais realizado porque tu tá vendo a tua produção, não fica escondido." (E11)

Considerando-se esse contexto, pode-se perceber que as falas que apontam a importância da SAE, mesmo que não completamente conceituada, aparecendo também justificativas para sua não realização, com comentários que descrevem a enfermagem como uma prática com foco técnico, tarefeiro e burocrático. Ressalta-se que é necessário salientar que muito mais do que uma sequência de etapas a ser seguida, mas um processo que exige conhecimento e habilidade na tomada de decisão do enfermeiro.

Estudo anterior referente às etapas da sistematização identificou que $52 \%$ dos profissionais apresentou respostas incompletas sobre suas etapas e $31,5 \%$ conseguiu descrevê-la completamente $^{(15)}$.

Para que a SAE seja efetivada em uma instituição de saúde, é necessário que os enfermeiros que lá atuam compreendam a enfermagem como ciência e que utilizem o conhecimento teórico que proporcione um direcionamento científico, promovendo um atendimento lógico, organizado e estruturado e implementado de estruturas que permitam progredir à organização do processo de cuidar ${ }^{(16)}$.

Na realização da SAE é imperativo o conhecimento das etapas do PE, uma vez que este fundamenta um importante instrumento para o cuidado sistematizado, uma vez que o mesmo norteia o cuidado. A opção de solucionar os problemas por tentativa de erro, certamente será um método sem êxito e perigoso para o paciente ${ }^{(17)}$.

Ao questionar os enfermeiros em 
relação às etapas do PE os informantes não demonstraram total familiaridade.

"Etapas da SAE é: planejamento (...) eu sei que é planejamento (...) Não estou me lembrando agora no momento. Não vou mentir." (E14).

"Histórico, anamnese, exame físico, as três que eu gravo que é o que eu tenho que fazer" (E16).

"Sei que tem o histórico, o diagnóstico (...) vou ter que me lembrar (...) avaliação eu acho (...) agora eu não me lembro de mais de todos" (E6).

Percebem-se, novamente, dúvidas ou desconhecimento teórico. O PE é um recurso que possibilita enfermeiros e pacientes trabalharem juntos em prevenção de doenças e enfermidades, recuperação da saúde e enfrentamento de funções alteradas, norteando a tomada de decisões e facilitando a percepção do problema, os diagnósticos e as prováveis intervenções com avaliação centrada nos resultados do cuidado com paciente ${ }^{(17,18)}$.

Os dados permitem inferir que, sobre a conceituação da SAE e as etapas que a envolvem, os entrevistados possuem conhecimento insuficiente ou equivocado. Mesmo refletindo sobre a importância desta ferramenta para a enfermagem, não percebem que sendo uma atividade exclusiva do enfermeiro, isso os diferencia. Muitas vezes justificam-se por meio de apelos burocráticos ou do tempo despendido na realização das atividades inerentes à profissão. Os mesmos argumentos refletem-se nas respostas aos questionamentos relacionados às etapas do $\mathrm{PE}$.

Nesse sentido, é importante a reflexão de que a SAE precisa ser discutida e valorizada, primeiramente, pelos profissionais da área de enfermagem. No entanto, é necessário promover alternativas que viabilizem esse trabalho, além da conscientização em relação ao seu papel de líder, o que pressupõe que o profissional tem capacidade e autonomia de transformar a de assistência à saúde.

Prática profissional: dificuldades encontradas

Da mesma forma que o conhecimento sobre a SAE e o PE são importantes e suas fases são imprescindíveis para sua execução, é necessário entender o que interfere na aplicabilidade da mesma no cotidiano dos enfermeiros. Percebeu-se que alguns enfermeiros negaram sua utilização, indicando uma assistência distanciada da fundamentação teórica:

"A gente tenta utilizar, mas às vezes é tanta correria que a gente faz o básico e acaba esquecendo mais o objetivo (...) mas eu acredito que eu tento usar um pouco (...) mas a gente não usa fidedigno como deveria ser" (E17.)

"A gente tenta utilizar, mas não consegue todas as etapas" (E1).

Sendo a SAE considerada um modelo de cuidado embasado em método científico que sistematiza e direciona o trabalho da equipe de enfermagem, salienta-se a necessidade de utilização de todas as etapas para que sejam alcançados os objetivos, incluindo o de atender as necessidades do paciente com habilidades, conhecimentos e atitudes alicerçados em compromisso ético e responsabilidade ${ }^{(19)}$.

Dos enfermeiros entrevistados e questionados quanto às dificuldades para realização da SAE em seu cotidiano, houve menção à falta de tempo como principal obstáculo. Os enfermeiros apontaram que, devido à demanda da atividade vinculada à as- sistência, não conseguem desenvolver completamente a SAE.

"Na verdade, a gente teria que utilizar em todas as etapas, mas a gente não consegue fazer que funcionem todas. A gente faz pelo menos a anamnese e parte da evolução (...)A evolução teria que fazer diariamente, só que infelizmente a nossa realidade não é bem essa. Mas a gente tenta fazer o máximo que a gente consegue" (E15).

“(...) pela falta de tempo (...) são muitos pacientes. Alguns precisam de mais atenção (...) a gente não consegue sentar e fazer tudo sozinha [...]" (E1).

A falta de tempo, dificuldades com dimensionamento de pessoal, a dificuldade no estabelecimento de prioridades na organização do processo de trabalho, assim como a cultura de desvalorização das prescrições de enfermagem são elementos estudados e publicados acerca das dificuldades para implementação e execução da $\operatorname{SAE}^{(13)}$.

Também são citadas como obstáculos: a estrutura do processo, como abrangência da coleta de dados, determinação das subfases do planejamento e falta de instrumento para avaliar os resultados mediante aos cuidados prestados; o cenário de ensino aprendizagem, compreendendo que esses processos devem exigir uma abordagem contínua ao longo da formação do aluno; e, por último, a compreensão, pelos profissionais de saúde, que o $\mathrm{PE}$ faz parte de uma rotina sofisticada e que colocá-lo em prática envolve distanciar-se do paciente ${ }^{(14)}$.

Devido à grande demanda de tarefas e sobrecarga de trabalho, entende-se pelos dados que, há necessidade de priorizar atividades. No entanto, ainda existe a representação de que a SAE é um procedimento desacredi- 
tado na sua prática, mesmo com reconhecimento de eixo norteador cuidados e exclusiva do enfermeiro ${ }^{(20)}$, havendo priorização de questões mais urgentes, não fundamentadas em um modelo assistencial.

Ao analisar as respostas, percebe-se que as dificuldades dos enfermeiros em aplicar a SAE não se limitam somente à prática profissional, mas também a falta de conhecimento efetivo sobre o tema.

"Ainda se eu soubesse o contexto todo, seria mais fácil dizer eu consigo realizar tranquila essa etapa e a outra não, mas como eu não tenho o conhecimento de todas elas eu não tenho como te explicar o que é dificuldade ou não" (E17).

"[...] Tem que haver um treinamento específico pra gente poder desenvolver um trabalho com melhor qualidade." (E18).

Quando há carência de conhecimento do processo de forma integral, ou a realização fragmentada de suas etapas, pode haver prejuízo do estímulo profissional ou debilitação da integração das etapas, dificultando assim o planejamento do cuidado ao paciente ${ }^{(21)}$. Enfatiza-se, mais uma vez, a necessidade de inserir o processo como uma metodologia integrada à rotina de enfermagem de forma sistemática ${ }^{(22)}$.

Quando questionados sobre as vantagens e desvantagens de realização sistematização, verificou-se que as vantagens são relacionadas à melhoria na qualidade dos cuidados dispensados aos pacientes e as desvantagens às interferências no processo de trabalho.

"A vantagem é que tu conhece o paciente por completo e faz uma avaliação diária de como ele tá evoluindo e progredindo. Desvantagem eu não vejo que tenha, só vantagens mesmo" (E9).

"A desvantagem é o nosso tempo, fazer tudo que uma enfermeira faz, (...) a parte administrativa (...) a enfermeira cuida de tudo (...) envolve muita coisa, é bem complicado" (E12).

Ao mesmo tempo em que os enfermeiros atribuem vantagens, acrescentam condições reais em seu cotidiano que podem favorecer o desenvolvimento de um trabalho deficiente, como a existência de muitas tarefas e a falta de tempo de observar tudo, mesmo considerando importante a prática da SAE.

Assim como já relatado na literatura, são encontrados relatos relacionados a vantagens e desvantagens. Destaca-se que as vantagens geralmente são relacionadas à qualificação e à qualidade da assistência prestada ${ }^{(23,24)}$. Apesar de todos os benefícios relacionados à $\mathrm{SAE}$, muitas têm sido as barreiras que dificultam esse processo em meio à sua complexidade e dimensão, como número reduzido de funcionários, sobrecarga de trabalho falta de capacitação da equipe e formação acadêmica inadequada, sem vínculo entre teoria e prática. Além disso, há resistência por parte dos profissionais, provavelmente relacionada ao desconhecimento sobre o tema, bem como baixo investimento por parte das instituições em treinamentos relacionados a esse tema ${ }^{(25)}$.

Sendo a SAE um dos principais instrumentos para o desenvolvimento da competência clínica do enfermeiro, é fundamental que estes profissionais busquem capacitação no intuito de ampliar seu conhecimento e campo de visão, não se limitando as práticas e demandas cotidianas, bem como o conhecimento adquiridos nos bancos acadêmicos.

Com base nessas análises, pode- 
mos estabelecer um contraponto: de um lado o reconhecimento de que a SAE é importante e que não há desvantagens na implementação na prática diária e de outro, a grande dificuldade relatada para não implementar devido à falta de tempo e falta de conhecimento teórico sobre o PE. É preocupante o fato de não haver uma metodologia assistencial estabelecida em alguns serviços de saúde, uma vez que sabe-se que a assistência pode ser prejudicada em sua qualidade e segurança, além da prática diária correr o risco de tornar-se desorganizada e levar à desvalorização e desgaste da equipe de enfermagem.

\section{CONCLUSÃO}

Percebe-se que, apesar das bases científicas comprovadas e as legislações sobre implementação da SAE, há um longo caminho a ser percorrido para que se torne uma realidade em todas as instituições de saúde.

A implementação da SAE deve ser realizada através de um processo lento e gradual, sendo indispensável superar obstáculos como a descrença no processo e a mudança de paradigmas na forma de ser e de compreender o papel do enfermeiro em sua prática assistencial.

As limitações que dificultam a implementação da SAE são persistentes em diferentes cenários, emergindo neste estudo, além dos relatos sobre falta de tempo, a carência no conhecimento científico. No entanto, cabe ao enfermeiro a busca pelo aprimoramento científico mesmo após a conclusão da formação acadêmica na graduação. O profissional precisa exercer uma prática que destaque a sua competência, bem como desenvolver atitudes e habilidades que são direcionadas a um cuidado com compromisso ético e responsabilidade.

A enfermagem depara-se com muitos desafios, contudo, é preciso somar os esforços para que a SAE se torne uma realidade nas instituições. Destaca-se a necessidade de difundir a SAE como método de trabalho, promover a educação continuada desses profissionais, reestruturar o quantitativo de pessoal e elaborar protocolos assistenciais na busca constante de qualificação profissional, que refletirão de maneira direta na qualidade e segurança assistencial prestada.

\section{Referências}

1. Souza MFG. Sistematização da Assistência de Enfermagem em um hospital pediátrico de ensino: uma pesquisa-ação. [Dissertação]. Natal (RN): Universidade Federal do Rio Grande do Norte; 2010 [Internet]. [citado 2015 Jun 24] Disponível em: http://repositorio.ufrn.br:8080/jspui/handle/123456789/14710 2. Mendes NPN. Conhecimento dos enfermeiros do hospital universitário Onofre Lopes sobre Sistematização da Assistência de Enfermagem. [Dissertação]. Natal (RN): Universidade Federal do Rio Grande do Norte; 2010 [Internet]. [citado 2015 Jun 24] Disponível em: http://www.repositorio.ufrn. br:8080/jspui/handle/123456789/14699

3. Hermida PMV, Araújo IEM. Sistematização da Assistência de Enfermagem: subsídios para implantação. Rev Bras Enferm. 2006; 59(5):675-9.

4. Conselho Federal de Enfermagem (COFEN). Resolução n. ${ }^{\circ} 272$, de 27 de agosto de 2002. Dispõe sobre a Sistematização da Assistência de Enfermagem - SAE - nas Instituições de Saúde brasileiras. Boletim COFEN normas e notícias 2002; 22:4-5.

5. Hermida PMV. Desvelando a implementação da Sistematização da Assistência de Enfermagem. Rev Bras de Enferm. 2004; 57(6):733-7.

6. Silva EGC, et al. 0 conhecimento do enfermeiro sobre a Sistematização da Assistência de Enfermagem: da teoria à prática. Rev Esc Enferm USP. 2011; 45(6):1380-6.

7. Conselho Regional de Enfermagem de São Paulo (COREN-SP). Anotações de Enfermagem. São Paulo: CORENSP; 2009.

8.Poter PA, Perry AG. Fundamentos de Enfermagem. 6. ed. Rio de Janeiro: Elsevier; 2005.

9.Polit DF, Beck CT, Hungler BP. Fundamentos de pesquisa em enfermagem: métodos, avaliação e utilização. 5. ed. Porto Alegre: Artes Médicas; 2004.

10.Minayo MCS, et al. Pesquisa social: teoria, método e criatividade. 11. ed. Petrópolis: Vozes; 2000.

11.Brasil. Lei n. ${ }^{\circ} 9610$, de 19 de fevereiro de 1998. Altera, atualiza e consolida a legislação sobre direitos autorais e dá outras providências. Diário Oficial da União: 20 de fev 1998.

12. Machado MH, Filho WA, Lacerda WF, Oliveira $E$, Lemos W, Wermelinger $M$ al. General characteristics of nursing: the sociodemographic profile. Enferm foco. 2016; 7(esp)11-7.

13. Remizoski J, rocha MM, Vall J. Dificuldades na implantação da sistemati- zação da assistência de enfermagem - SAE: umarevisão teórica. Cadernos da escola de saúde. 2010; 3:1-14.

14. Carvalho EC, Bachion MM, Dalri MCB, Jesus CAC. Obstáculos para a implementação do processo de Enfermagem no Brasil. Rev Enferm UFPE. 2007; 1(1):95-9. 15. Silva LWS, Nunes ECDA, Souza DM, Santos CS, Pereira LM. Sistematização da Assistência de Enfermagem: a práxis no ser-saber-fazer o cuidado. Cogitare Enferm 2011;16(3).

16. Kroger MMA, Bianchini SM, Oliveira AML, Santos LSC. Enfermagem em Terapia Intensiva: do ambiente da unidade à assistência ao paciente. São Paulo: Martinari; 2010.

17. Taylor C, Lillis C, Lemone P. Fundamentos de Enfermagem. 5. ed. Porto Alegre: Artmed; 2007.

18. Figueiredo MED, Santos SR, Oliveira AMM,Leite NS, Morais JMD, Duarte ACP. Sistematização da Assistência de Enfermagem: percepção de enfermeiros de um hospital escola. Rev Enferm UFPE. 2013; 7(esp):6981-8.

19. Menezes SRT, Priel MR, Pereira LL. Autonomia e vulnerabilidade do enfermeiro na prática da Sistematização da Assistência de Enfermagem. Rev Esc Enferm USP. 2011; 45(4):953-8.

20. Costa AC, Silva JV. Representações sociais da sistematização da assistência de enfermagem sob a ótica de enfermeiros. Rev. Enf. Ref. 2018; IV(16):139-46. 21. Oliveira KF, Iwamoto HH, Oliveira JF, Almeida DV. Sistematização da Assistência de Enfermagem na Rede Hospitalar de Uberaba: MG. Rev. Enf. Ref. 2012; 3(8):105-14.

22. Cavalcante RB, Otoni A, Bernardes MFVG, Cunha SGS, Santos CS, Silva PC. Experiências de Sistematização da Assistência de Enfermagem no Brasil: um estudo bibliográfico. Rev Enferm UFSM. 2011; 1(3):461-71. 23. Moreira V, Santos CS, Oliveira JC, Reis LA, Lima EF. Sistematização da Assistência de Enfermagem: desafios na sua implantação. Inter Scientia. 2013; 1(3):60-79.

24. Freire EMR, Carvalho CC, Resck ZMR. Sistematização da assistência de enfermagem no processo de trabalho hospitalar: uma revisão integrativa. Revista Eletrônica Acervo Saúde. 2012; 4(2):308-26.

25. Dias IMAV, Terra AAA, Machado JRO, Reis VN. Sistematização da Assistência de Enfermagem no gerenciamento da qualidade em saúde. Rev Baia de Enferm. 2011; 25(2):161-72. 